\title{
When Coordination and Exploration are matched: A Simulation Research on Multi-Agents Governance mode
}

\author{
Cunying Chen ${ }^{1, a}$, Hua Zhang ${ }^{2, b}$ \\ ${ }^{1}$ Information Construction and Management Office, Hua Qiao University, Quanzhou 362021, China; \\ ${ }^{2}$ College of Business Administration, Hua Qiao University, Quanzhou 362021, China. \\ achencunying@hqu.edu.cn, bzhanghuav@hqu.edu.cn
}

Keywords: Computational Complexity Theory, Object-oriented Programming, Simulation.

\begin{abstract}
Engaging in exploring multi-agent collaboration requires determining how to govern the shared activities. We examine the performance implications of selecting alternate modes of governance in multi-agent alliance relationships. A core set of results in this study relates to the ways in which governance structure interacts with agents' search capabilities. Alliance performance improves when the needs and supplies of coordination and exploration are matched. We find situations in which there is an inverted $U$ relationship between governance mode and performance. At the end we discuss the contribution and the possibility of extension in the future.
\end{abstract}

\section{Introduction}

Multi-agent collaborative relationships are a growing phenomenon with significant performance consequences [1][2]. It became more and more popular in computational complexity theory, object-oriented programming and other related research field. Engaging in exploring such multi-agent collaboration requires determining how to govern the shared activities - a particularly important consideration when the agents are connected in a particular way, as an alliance. Although many scholars have admitted that different governance mode choice would lead to different kind of strategic implications [3][4], there is limited direct evidence for the impact of different modes on agents' alliance performance. Furthermore, there is still an open question that what factors compel agents to select alternate modes of governance in cooperative settings? According to the prior research, two streams of prior work have addressed the determinants of agents' alliance governance: a transaction cost economics (TCE) view, which generally conclude that the reason why agents choose a certain governance mode is determined by the coordination cost [5]; and an computational complexity simulation approach, which taking the different agent's exploration ability into account when examining the consequent performance [6]. In this study we try to combine these two theory view. We argue that there should be a balance between exploration and coordination when we explore the mechanism under the situation where multi-agents collaborate with each other facing a parallel problem. To examine this question, we employ an agent-based simulation that enables us to develop a more nuanced view of the consequence of governance modes. Particularly, we take a simple alliance case for our simulation circumstance: a triple agent alliance where three agents are connected in a line. To anchor our research expectation we first construct a multi-agent alliance model that builds on the agents' information processing ability and computational complexity theory. With this computational model we then use the simulation to generate further insight into the mechanisms the alternate governance modes effect on performance.

\section{Model construction}

\subsection{Problem space.}

To develop our simulation model, we build on and extend prior work that has used the NK framework to model multi-agent decision making and external environment [7][8]. In particular, the triple alliance is facing a problem space which includes $\mathrm{N}$ decision choice. The NK model further 
assumes that the performance is not only determined by the self-decision choice but also by $\mathrm{K}$ other choice. In sum, each unique configuration of $\mathrm{N}$ choices leads to a performance value. We can imagine a performance landscape on $\mathrm{N}+1$ dimensions: the horizontal dimension is including $\mathrm{N}+1$ choice and the vertical dimension is the performance value. Landscape complexity depends on interactions among $\mathrm{K}$ choices: when $\mathrm{K}=0$, we have a flat landscape while $\mathrm{K}$ is approaching $\mathrm{N}$ we got a rugged landscape.
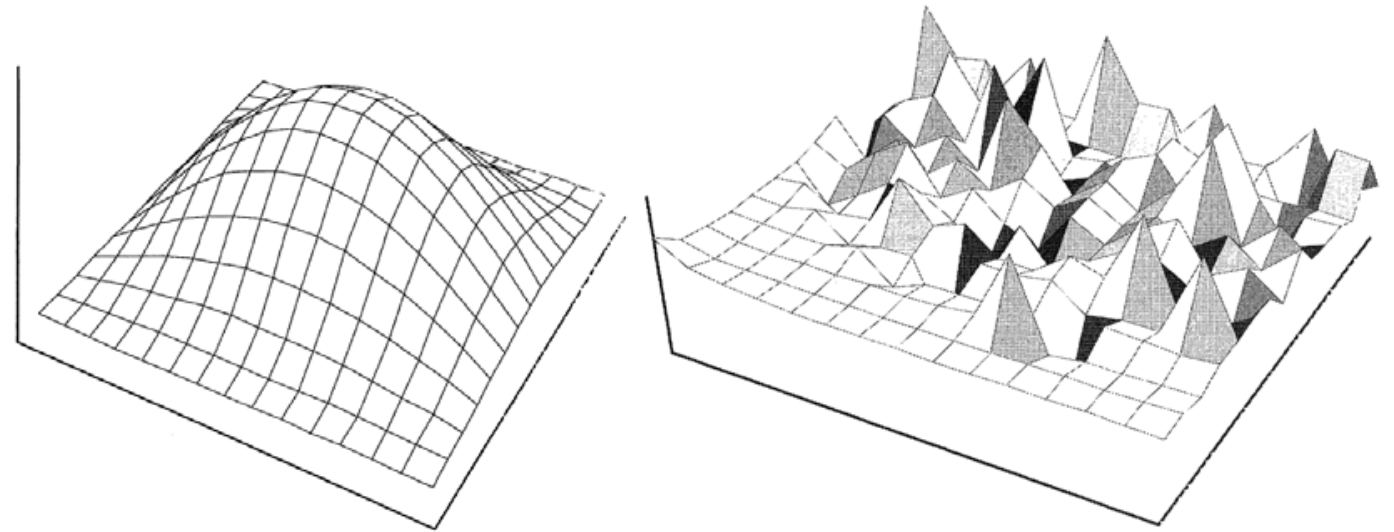

Fig.1 Flat and Rugged Landscape.

We model three agents collaborate with each other to search the solution on a $\mathrm{N}=16$ problem space. Specially, we denote agent $\mathrm{A}, \mathrm{B}$ and $\mathrm{C}$, agent $\mathrm{A}$ and $\mathrm{C}$ both charge 4 decision choices but agent $\mathrm{B}$ who hold a broker position is responsible for 8 choices. Because of none of them search on the whole problem space they should collaborate with each other and combine their solutions to a unique one to achieve alliance performance.

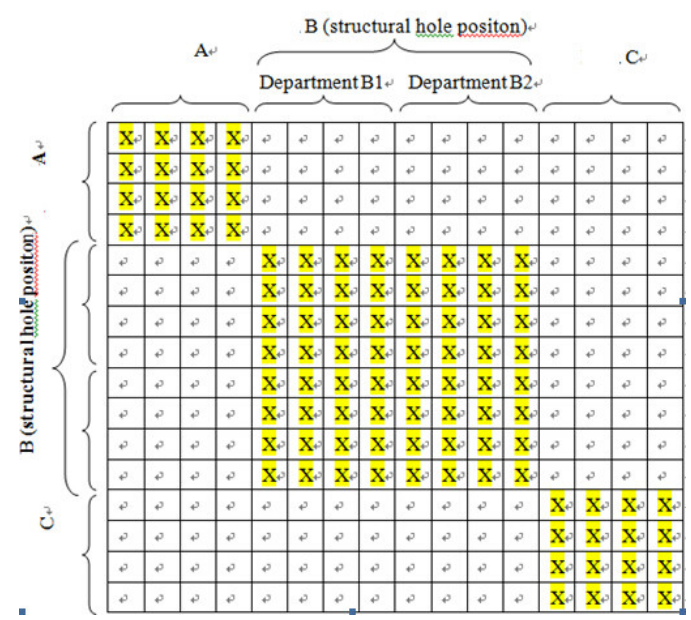

Fig.2 job assignment

\subsection{Agents search capabilities.}

We turn now to the decision-making rules that govern agents' behavior in our simulation. Basing on the prior search [9][10], we model two dimensions of exploration capabilities: (1) the ability to make simultaneous decisions over a larger vs. smaller number of the choices controlled, which we term the 'search radius' as per prior literature [11]; and (2) the ability to evaluate a larger vs. smaller number of alternatives in a given period [12]. For the search radius (parameter SR), we model a simple case where $\mathrm{SR}=2$, as well as a more complex case where $\mathrm{SR}=4$; for the number of alternatives (parameter ALT), we also model a simple case where ALT=4 and a complex case where ALT=14.

\subsection{Governance modes}

There are four governance modes in our model: Modular, MOD, this mode focuses on sub-project optimal and search arrange are A,C, B1 and B2; Corporation, COP, under this mode agent only consider their own decision choices; Department, DEP, this mode divide the whole alliance into two part and the search range are $\mathrm{A}+\mathrm{B} 1$ and $\mathrm{B} 2+\mathrm{C}$; Integration, INT, this mode focuses on integrating the three agent's searching part. 


\section{Simulation experiment design}

In the case of $\mathrm{N}=16$ the configuration contain a total of 65,536 decisions to select. We use parameter $\mathrm{K}$ to control the external environment, SR and corresponding ALT control agents' exploration capabilities. And we explore how the four governance modes (represent for coordination) affect the final performance. Each simulation experiment is set to 300 time steps, after repeated experiments estimated 300 time steps after the system has reached equilibrium, Alliance Performance Management Mode four have reached their highest point. Each experiment will be repeated 1000 times and the results averaged to eliminate the influence of random errors.

\section{Results and Analysis}

When $K=1$, because of the simple environment every governance mode can easily get to the best performance at the end, however, they show a different trajectory picture. As showed in figure 4, when $\mathrm{SR}=2$; $\mathrm{ALT}=4$, although the four governance modes achieve the same performance level at the end, INT and COP show the classic trade-off picture of short-long run performance: in the short run COP get the higher performance while in the long run INT can get the same performance level. For broker agent, "Divide and Conquer" strategy (COP) can promote performance quickly, but for the long run "Unite” strategy will show its advantage.

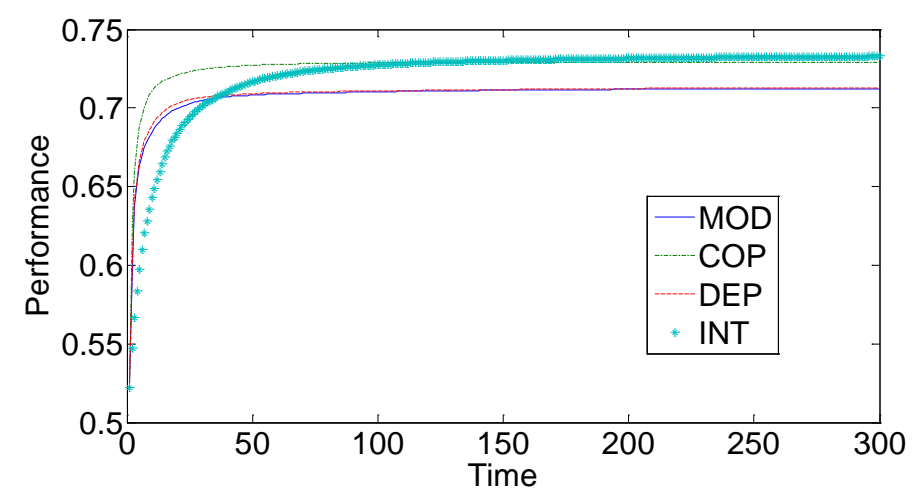

Fig 4 Relationship between governance and performance (simple environment and poor ability)

Finally we model agents' strong ability. Fig 5 show us that the most important factor influencing the alliance performance is the coordination rang which has an inverse $U$ relationship with performance. The highest performance is under the governance of DEP which has a medium coordination rang, while the high (INT) or low(MOD and DEP) coordination rang achieve the lower performance. This result also prove the importance of balance between exploration rang and coordination cost. The medium mode surpass the others.

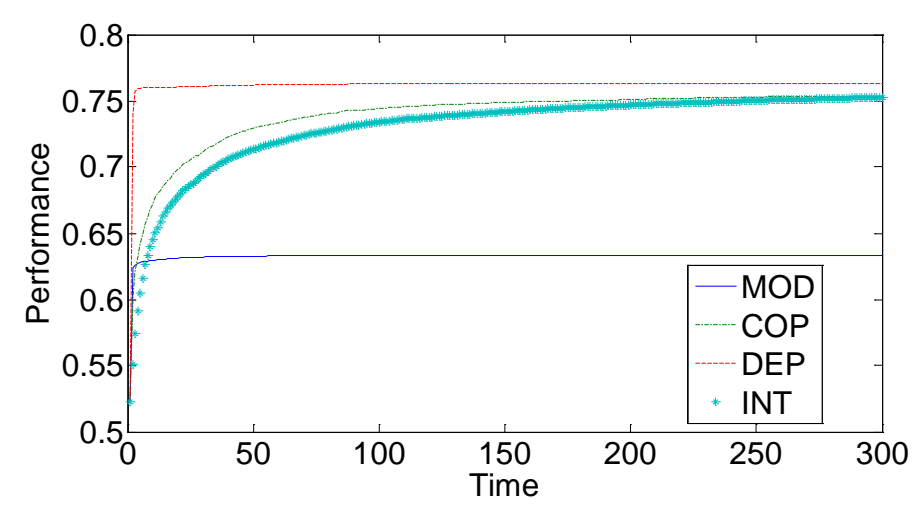

Fig 5 Relationship between governance and performance (simple environment and poor ability)

\section{Conclusion and discussion}

In this study, we conduct a simulaiton experiment to explore how four governance modes affact alliance performance. When agents have weak exploration ability, we should pay more attention on 
the performance trade-off between exploration range and governance model cost: in the short term structural holes agent who focus itself would promote performance, but in long term this strategy would not be sustainable. A full exploration of the problem domain Governance will bring a league-high performance. The most typical case of the match among these three dimension is under the situation of stronge exploration ability. When structural holes agent advocate quasi-market governance (DEP) the alliance performance would be the best. This conclusion have parallels with results from prior research Aggarwal.V (2011) [13] whoes study advocated alliances governance and coordination meet the balance of demand and supply of exploration and coordination.

The results of our simulation research fully demonstrate the importance of the balance of exploration ability and coordination governance. None of governance mode achieves a unique and permanent advantage. We should take into account of exploration ability, external environment and governance mode when searching for the high performance level. As a fundamental simulation model there is absolutely space to expand in the future research. Such like add directed relationship among agents, more subgroup design and so on [14][15]. The author encourages others to apply a multi-agents lens to studies of alliance performance in comparative fields in order to broaden the complexity and simulation research.

\section{References}

[1] Xiao Z, Tsui A S. When Brokers May not Work: The Cultural Contingency of Social Capital in Chinese High-Tech Firms[J]. Administrative Science Quarterly. 2007, 52(1): 1-31.

[2] Obstfeld D. Social Networks, the Tertius Iungens Orientation, and Involvement in Innovation[J]. Administrative Science Quarterly. 2005, 50(1): 100-130.

[3] Lichtenthaler U, Lichtenthaler E. Alliance functions: implications of the international multi-R\&D-alliance perspective[J]. Technovation. 2004, 24(7): 541-552.

[4] Hoang, H. and F.T. Rothaermel, The effect of general and partner-specific alliance experience on joint R\&D project performance. Academy of Management Journal, 2005. 48(2): p. 332-345.

[5] Williamson, O.E., The Economics of Organization: The Transaction Cost Approach. American Journal of Sociology, 1981: p. 548-577.

[6] Gulati, R. and H. Singh, The architecture of cooperation: Managing coordination costs and appropriation concerns in strategic alliances. Administrative science quarterly, 1998: p. 781-814.

[7] Siggelkow, N. and J.W. Rivkin, Speed and search: Designing organizations for turbulence and complexity. Organization Science, 2005. 16(2): p. 101-122.

[8] Kale, P., J.H. Dyer, and H. Singh, Alliance capability, stock market response, and long - term alliance success: the role of the alliance function. Strategic Management Journal, 2002. 23(8): p. 747-767.

[9] Sampson, R.C., The cost of misaligned governance in R\&D alliances. Journal of Law, Economics, and Organization, 2004. 20(2): p. 484-526.

[10] Davis J P, Eisenhardt K M, Bingham C B. Developing Theory Through Simulation Methods[J]. The Academy of Management Review, 2007, 32(2): 480-499.

[11] Wright S. The roles of mutation, inbreeding, crossbreeding and selection in evolution [C]. Proceedings of the VI International Congress of Genetrics, 1932,1(1):356-366

[12] Kauffman S A. Cellular Homeostasis, Epigenesis and Replication in Randomly Aggregated Macromolecular Systems[J]. Cybernetics and Systems, 1971, 1(1): 71-96.

[13] Aggarwal V A, Siggelkow N, Singh H. Governing collaborative activity: interdependence and the impact of coordination and exploration[J]. Strategic Management Journal. 2011, 32(7): 705-730.

[14] Levinthal D A. Adaptation on Rugged Landscapes[J]. Management Science, 1997, 43(7): 934-950. 
[15] Fang C, Lee J, Schilling M A. Balancing Exploration and Exploitation Through Structural Design: The Isolation of Subgroups and Organizational Learning[J]. Organization Science. 2010, 21(3): 625-642. 